OPEN ACCESS

Edited by:

Joseph Curry

Thomas Jefferson University,

United States

Reviewed by:

Christos K. Kontos,

National and Kapodistrian University

of Athens, Greece

Amilcare Barca,

University of Salento, Italy

${ }^{*}$ Correspondence:

Ting Zhang

ztcathy_05@163.com

Yong Mao

mydoctor@aliyun.com

Dong Hua

wx89211@163.com

Specialty section:

This article was submitted to

Cancer Metabolism,

a section of the journal

Frontiers in Oncology

Received: 29 April 2020

Accepted: 20 August 2020

Published: 15 September 2020

Citation:

Zhang T, Jin Y, Jiang X, Li L, Qi X,

Mao Y and Hua D (2020) Clinical and Prognostic Relevance of $\mathrm{B} 7-\mathrm{H} 3$ and Indicators of Glucose Metabolism

in Colorectal Cancer.

Front. Oncol. 10:546110.

doi: 10.3389/fonc. 2020.546110

\section{Clinical and Prognostic Relevance of B7-H3 and Indicators of Glucose Metabolism in Colorectal Cancer}

\author{
Ting Zhang ${ }^{1 *}$, Yufen $\mathrm{Jin}^{2}$, Xin Jiang ${ }^{2}$, Longhai $\mathrm{Li}^{2}$, Xiaowei Qi ${ }^{2,3}$, Yong Mao ${ }^{2,4 *}$ and \\ Dong Hua ${ }^{1,2,4 *}$
}

${ }^{1}$ Institue of Cancer, Affiliated Hospital of Jiangnan University, Wuxi, China, ${ }^{2}$ Wuxi School of Medicine, Jiangnan University, Wuxi, China, ${ }^{3}$ Department of Pathology, Affiliated Hospital of Jiangnan University, Wuxi, China, ${ }^{4}$ Department of Oncology, Affiliated Hospital of Jiangnan University, Wuxi, China

Objective: This study aimed to investigate the clinical and prognostic relevance of B7$\mathrm{H} 3$ expression and indicators of glucose metabolism in patients with colorectal cancer (CRC).

Methods: Using immunohistochemistry, the expression of $\mathrm{B} 7-\mathrm{H} 3$ was detected in a total of 213 formalin-fixed paraffin-embedded CRC tissue specimens. Furthermore, levels of fasting blood glucose (FBG), lactic dehydrogenase (LDH), and fructosamine (FMN) as indicators of glucose metabolism were analyzed in CRC patients and stratified into high or low expression sub-groups based on Youden Index. The relationship between B7-H3, FBG, LDH, FMN expression, and clinicopathological characteristics were also evaluated to establish their prognostic significance in patients with $\mathrm{CRC}$.

Results: $\mathrm{B} 7-\mathrm{H} 3$ was highly expressed in $\mathrm{CRC}$ tissue. The positive rates of $\mathrm{B} 7-\mathrm{H} 3$ expression was 63.8\% (136/213). We found a linear correlation between B7-H3 and FBG in depth of tumor invasion (T3/4) $(p=0.037, r=0.259)$, lymph node metastasis (NO) $(p=0.004, r=0.259)$, and TNM stage $(I / I)(p=0.009, r=0.242)$. High expression of FBG, LDH, FMN [hazard ratio $(\mathrm{HR})=1.916,95 \% \mathrm{Cl}: 1.223-3.00, p=0.005 ; \mathrm{HR}=1.801$, 95\% Cl: 1.153-2.813, $p=0.010 ; \mathrm{HR}=2.154,95 \% \mathrm{Cl}: 1.336-3.472, p=0.002]$, respectively, was identified as a significant independent predictor of poor overall survival (OS). Although B7-H3 expression did not affect OS, CRC patients expressing both high $\mathrm{B} 7-\mathrm{H} 3$ and high FMN contributed to a significant decrease in OS $(\mathrm{HR}=1.881,95 \% \mathrm{Cl}$ : 1.059-3.339, $p=0.031$ ). Moreover, with low expression of $\mathrm{B} 7-\mathrm{H} 3$, high expression of FBG, LDH and FMN were also recognized as predictors of inferior OS $(H R=3.393,95 \%$ Cl: 1.493-7.709, $p=0.004 ; \mathrm{HR}=7.107,95 \% \mathrm{Cl}: 2.785-18.138, p=0.000 ; \mathrm{HR}=2.800$, $95 \% \mathrm{Cl}: 1.184-6.625, p=0.019)$.

Conclusion: B7-H3 combined with FBG, LDH, or FMN, could reflect the clinical outcomes of patients with CRC.

Keywords: B7-H3, glucose metabolism, colorectal cancer, correlation, prognosis 


\section{INTRODUCTION}

Colorectal cancer (CRC) remains the third most frequently diagnosed gastrointestinal tract malignancy in men and second in women, globally. Although the mortality rate of CRC has been declining progressively, it still represents the third leading cause of cancer-associated mortality for both men and women (1). In contrast to these declines, CRC remains the most rapidly increasing cancer in China over the past few decades. This rise in CRC incidence has become one of the major public health concerns causing a substantial health burden to families and also contributing to overburdened healthcare systems (2). Recently, Gu et al. summarized tobacco smoking, alcohol abuse, obesity, low physical activity, low fruit and fiber consumption, and high intake of red and processed meat as the significant attributable causes of CRC in China (3). Moreover, these risk factors were also associated with poor prognosis of CRC, particularly in CRC patients with diabetes mellitus (DM).

In the past three decades, the prevalence of DM and associated health complications has substantially increased in the world, and DM represented the ninth leading cause of death (4). Notably, China has rapidly emerged as a region with DM global epidemic, owing to an unhealthy diet and a sedentary lifestyle as critical drivers (5). Moreover, accumulating epidemiological studies have indicated a positive association of DM with the risk of CRC (6). Several similar risk factors, including western lifestyle, have been reported between DM and CRC. Furthermore, fasting blood glucose (FBG), as an indicator of DM, was also found to be significantly related to the risk of CRC (7). Also, aerobic glycolysis is the most common energy metabolism characteristic of cancer cells. Previously, Graziano et al. analyzed mRNA expression of the key enzymes including GLUT1, LDHA, HK1, PKM2, and VDAC1 mRNA involved in glycolysis in CRC patients and revealed that expression levels were significantly higher in primary tumor tissues compared with normal mucosa (8). Besides, altered expression of genes involved in glucose uptake and glycolysis was also reported in CRC tissues $(9,10)$.

Immune checkpoint protein B7 homolog 3 (B7-H3 or CD276), a type I transmembrane glycoprotein, belongs to the B7 and CD28 superfamilies. While limited-expression has been observed in normal healthy tissues, overexpression of B7-H3 has been reported in a variety of malignancy and plays a crucial role in tumor progression (11). Clinically, B7-H3 overexpression in tumors has been associated with poor clinical outcomes (12). Moreover, increasing studies have indicated that the aberrant expression of $\mathrm{B} 7-\mathrm{H} 3$ is a consistent characteristic of CRC. Thus, B7-H3 detection might be an effective means to predict the prognosis in patients with CRC. Besides, Fang et al. demonstrated an increased level of soluble B7-H3 in type 1 diabetes patients compared with healthy controls (13), implying that $\mathrm{B} 7-\mathrm{H} 3$ might be a promising biomarker in the pathogenesis of diabetes. Furthermore, by inducing expression of the key glycolytic enzyme, hexokinase 2 (HK2), overexpression of $\mathrm{B} 7-\mathrm{H} 3$ has been documented to increase the rate of glucose consumption and lactate production
(14). These findings indicated that B7-H3 might be a novel regulator of glucose metabolism in CRC cells and a promising therapeutic target for CRC. Moreover, in hypoxic conditions, lactic dehydrogenase (LDH) has been known to convert pyruvate to lactic acid to support tumor cells, and elevated serum $\mathrm{LDH}$ has been reported to express in cancer and considered to be an independent prognostic predictor $(15,16)$. Besides, fructosamine (FMN) predominantly represents a measure of glycated albumin, which is the most abundant of the serum proteins. As the half-life of albumin is shorter than that of hemoglobin A1c (HbA1c), FMN reflects a shorter duration of glycemic control over the past 2-3 weeks period. This evidence indicates that FMN could be used as a short-term marker of glucose control. Moreover, elevated FMN levels have been associated with an increased risk of colorectal adenoma, a precursor of CRC (17).

Despite these pieces of evidence, only limited studies have been focused on B7-H3 and the indicators of glucose metabolism (FBG, LDH, and FMN) in CRC patients. Therefore, the present study was initiated to investigate the clinical and prognostic significance of B7-H3, FBG, LDH, and FMN in patients CRC.

\section{MATERIALS AND METHODS}

\section{Patients and Samples Collection}

The medical records of patients who received a histopathological diagnosis and underwent surgical resection for CRC at the Affiliated Hospital of Jiangnan University between June 2008 and December 2011 were included. A total of 213 formalinfixed paraffin-embedded CRC tissue samples were included in this study. For this study, only histopathologically confirmed cases were included. Besides, patients who received chemoor radiotherapy before surgery and cases with incomplete clinical data were excluded from the study. For all patients, pathological stages were defined according to the seventh edition American Joint Committee on Cancer (AJCC) cancer staging manual using available clinical and pathologic tumor, node, and metastasis data. Patient demographic data, including age and gender and clinicopathologic characteristics, such as tumor size and histologic grade and FBG, LDH, and FMN from clinical laboratory investigation, were also collected. This study was approved by the Medical Ethics Committee of the Affiliated Hospital of Jiangnan University (No. LS2019027), and written informed consent was obtained from all patients. All the patients were followed-up over telephone up to October 31, 2017, in order to analyze patient survival. The median follow-up was 80 months (range; 8-115 months).

\section{Tissue Microarray Construction and Immunohistochemistry Staining}

Two experienced pathologists examined the section stained with hematoxylin and eosin (HE) and marked the carcinoma sites in the corresponding paraffin block. The detailed tissue microarray (TMA) construction protocol followed was as described previously (18). Briefly, the TMAs were constructed by obtaining a tissue cylinder of $1.0-\mathrm{mm}$ in diameter core tissue 
biopsies from representative paraffin-embedded sections of each donor tissue block and implanted into the hole of the premade recipient paraffin block. The tissue paraffin blocks were serially sectioned into $4 \mu \mathrm{m}$ thick sections, deparaffinized in xylene, and hydrated in an ethanol gradient. Antigen retrieval was performed by heating the tissue sections at $100^{\circ} \mathrm{C}$ in sodium citrate buffer for $30 \mathrm{~min}$. Moreover, endogenous peroxidase was blocked by incubation in 3\% hydrogen peroxide for $10 \mathrm{~min}$. Subsequently, the sections were incubated in $5 \%$ bovine serum albumin (BSA) at room temperature (RT) for $30 \mathrm{~min}$. Primary antibody, mouse anti-human B7-H3 monoclonal antibody (1:200, Santa Cruz, CA, United States) added dropwise followed by overnight incubation at $4^{\circ} \mathrm{C}$. Following incubation, the slides were washed and incubated with a horseradish peroxidase-conjugated secondary antibody for $30 \mathrm{~min}$. The immunostaining was carried out by staining with 3, 3'-diaminobenzidine chromogen (DAB) (GTVisionII Immunohistochemistry Detection Kit for Rabbit/Mouse, GeneTech, China) and counter-stained with hematoxylin, dehydrated, and mounted and the sections were examined under a microscope. Two independent pathologists evaluated the percentage of positive cells (score of $0: \leq 5 \%$; score of 1: $6-25 \%$; score of 2: $26-50 \%$; score of 3: $51-75 \%$; score of 4 : $>76 \%$ ) and the intensity [0 for negative staining (no coloration); 1 for weakly positive (faint yellow); 2 for moderately positive (yellowish brown); 3 for strongly positive (brown)] of all samples. The two scores were multiplied to generate the final score for each specimen range from 0 to 12 .

\section{Verification of the Cut-Off Value}

We analyzed the receiver operating characteristics (ROC) curve of B7-H3, FBG, LDH, and FMN through SPSS 23.0 software (IBM, United States). Then, the Youden Index was calculated according to the formula sensitivity + specificity -1 . The maximum Youden Index representing the expression value of the target was considered as the cut-off value. Based on values, patients with less than cut-off value was defined as low expression group and those with a value equal or greater than cut-off value as described as high expression group.

\section{Statistical Analysis}

The relationship between clinicopathological parameters and B7H3, FBG, LDH, or FMN was analyzed using the Chi-square test. The Mann-Whitney test was used to analyze the difference of the expression of B7-H3, FBG, LDH, and FMN for each clinical characteristic. The non-parametric Spearman test evaluated the correlation of B7-H3 and FBG, LDH, or FMN expression in CRC patients. Overall survival (OS) was plotted using the KaplanMeier method and compared using the log-rank test. The Cox proportional hazard model was used to perform multivariate survival analysis. The $\mathrm{R}$ software version 3.6.1 and the RMS package ( $\mathrm{R}$ Foundation for Statistical Computing) were used to perform the nomogram analysis and calibration plot. All data were analyzed using the SPSS package (version 23.0, IBM, Chicago, IL, United States). All figures were generated with GraphPad Prism 6.0 (GraphPad Software Inc., United States). A $P$-value of $<0.05$ was considered statistically significant.

\section{RESULTS}

\section{Clinicopathological Parameters of Patients}

The clinicopathological parameters of 213 CRC patients were summarized in Table 1. The study comprised 118 males (55.4\%) and 95 females $(44.6 \%)$. The age at initial diagnosis was classified into $<60$ years $(37.6 \%)$ or $\geq 60$ years $(62.4 \%)$, and the median age of the patients was 62 (range, $27-87$ years old). $38.5 \%$ of tumors were located or distributed in the colon $(n=82)$ and $61.5 \%$ of tumors were located in the rectum $(n=131)$. Lymph node metastasis (N1-N2) was noted in $43.7 \%$ of cases. There were 115 (54.0\%) cases with stage I-II disease and 98 (46.0\%) cases with stage III-IV disease.

\section{Expression of B7-H3, FBG, LDH, and FMN in CRC Patients}

Using immunochemistry, we detected the expression of B7-H3 in CRC tissue. According to the cut-off value of 2.5, scores of 0-2 were defined as the low expression group, and scores of 3-12 were considered as the high expression group. Similar to previous studies, B7-H3 was highly expressed in CRC tissue. The positive rates of B7-H3 expression were 63.8\% (136/213). Furthermore, positive staining of $\mathrm{B} 7-\mathrm{H} 3$ was predominantly localized in the cytoplasm and membrane of tumor cells. The representative images were presented in Figure 1. Similarly, based on the value of FBG, LDH, and FMN from the clinical laboratory and survival outcome of patients, we calculated the cut-off value using the Youden Index. Thus, patients were defined as either low expression of FBG with the value $<5.425$ or high expression of FBG with the value $\geq 5.425$. We found that $32.9 \%$ of cases exhibited high expression of FBG (70/213). Also, the cutoff value of $\mathrm{LDH}$ and FMN were defined as 169.5 and 202.5, respectively, and the corresponding positive rates of $\mathrm{LDH}$ and FMN expression in patients with CRC were $35.7 \%(76 / 213)$ and $21.1 \%(45 / 213)$.

\section{Correlation Between B7-H3, FBG, LDH, and FMN and Clinicopathological Characteristics of Patients With CRC}

A significant difference between the expression of B7-H3 and the gender of patients with CRC was found $(p=0.028)$. Notably, positive expression of $\mathrm{LDH}$ was significantly associated with tumor location $(p=0.048)$ and distant metastasis $(p=0.009)$. Furthermore, the FMN expression was significantly related to the lymph node metastasis $(p=0.013)$ and the TNM stage $(p=0.014)$. However, the expression of FBG exhibited no significant association with any clinicopathological characteristics (Table 1). Using the Mann-Whitney test, we confirmed the marked differences between B7-H3 and gender $(p=0.046), \mathrm{LDH}$, and distant metastasis $(p=0.009)$. Notably, the expression of FMN was significantly associated with tumor location $(p=0.016)$, colon cancer site $(p=0.009)$, depth of tumor invasion $(p=0.032)$, lymph node metastasis $(p=0.046)$, and TNM stage $(p=0.033)$ (Figure 2). 
TABLE 1 | Association of B7-H3, FBG, LDH, and FMN expression in CRC patients with clinicopathological parameters.

\begin{tabular}{|c|c|c|c|c|c|c|c|c|c|c|c|c|c|}
\hline \multirow[t]{2}{*}{ Clinical parameter } & \multirow[t]{2}{*}{ Case $(n)$} & \multicolumn{2}{|c|}{ B7-H3 expression } & \multirow[t]{2}{*}{$p$ value } & \multicolumn{2}{|c|}{ FBG expression } & \multirow[t]{2}{*}{$p$ value } & \multicolumn{2}{|c|}{ LDH expression } & \multirow[t]{2}{*}{$p$ value } & \multicolumn{2}{|c|}{ FMN expression } & \multirow[t]{2}{*}{$p$ value } \\
\hline & & Low & High & & Low & High & & Low & High & & Low & High & \\
\hline Gender & & & & 0.028 & & & 0.216 & & & 0.545 & & & 0.718 \\
\hline Male & 118 & 35 & 83 & & 75 & 43 & & 78 & 40 & & 92 & 26 & \\
\hline Female & 95 & 42 & 53 & & 68 & 27 & & 59 & 36 & & 76 & 19 & \\
\hline Age (years) & & & & 0.572 & & & 0.831 & & & 0.872 & & & 0.467 \\
\hline$<60$ & 80 & 27 & 53 & & 53 & 27 & & 52 & 28 & & 61 & 19 & \\
\hline$\geq 60$ & 133 & 50 & 83 & & 90 & 43 & & 85 & 48 & & 107 & 26 & \\
\hline Tumor location & & & & 0.098 & & & 0.237 & & & 0.048 & & & 0.252 \\
\hline Colon & 82 & 24 & 58 & & 59 & 23 & & 46 & 36 & & 68 & 14 & \\
\hline Rectum & 131 & 53 & 78 & & 84 & 47 & & 91 & 40 & & 100 & 31 & \\
\hline Colon cancer site & & & & 0.371 & & & 0.655 & & & 0.763 & & & 0.212 \\
\hline Left-sided & 176 & 66 & 110 & & 117 & 59 & & 114 & 62 & & 136 & 40 & \\
\hline Right-sided & 37 & 11 & 26 & & 26 & 11 & & 23 & 14 & & 32 & 5 & \\
\hline \multicolumn{2}{|c|}{ Depth of tumor invasion } & & & 0.130 & & & 0.335 & & & 0.231 & & & 0.588 \\
\hline $\mathrm{T} 1 / 2$ & 64 & 28 & 36 & & 46 & 18 & & 45 & 19 & & 49 & 15 & \\
\hline T3/4 & 149 & 49 & 100 & & 97 & 52 & & 92 & 57 & & 119 & 30 & \\
\hline \multicolumn{2}{|c|}{ Lymph node metastasis } & & & 0.494 & & & 0.192 & & & 0.600 & & & 0.013 \\
\hline NO & 120 & 41 & 79 & & 85 & 35 & & 79 & 41 & & 102 & 18 & \\
\hline $\mathrm{N} 1 / 2$ & 93 & 36 & 57 & & 58 & 35 & & 58 & 35 & & 66 & 27 & \\
\hline \multicolumn{2}{|l|}{ Distant metastasis } & & & 0.428 & & & 0.968 & & & 0.009 & & & 0.063 \\
\hline Yes & 15 & 4 & 11 & & 10 & 5 & & 5 & 10 & & 9 & 6 & \\
\hline No & 198 & 73 & 125 & & 133 & 65 & & 132 & 66 & & 159 & 39 & \\
\hline \multicolumn{2}{|l|}{ TNM stage } & & & 0.870 & & & 0.161 & & & 0.384 & & & 0.014 \\
\hline$|/| \mid$ & 115 & 41 & 74 & & 82 & 33 & & 77 & 38 & & 98 & 17 & \\
\hline III/IV & 98 & 36 & 62 & & 61 & 37 & & 60 & 38 & & 70 & 28 & \\
\hline \multicolumn{2}{|l|}{ Neural invasion } & & & 0.685 & & & 0.288 & & & 0.479 & & & 0.917 \\
\hline Yes & 39 & 13 & 26 & & 29 & 10 & & 27 & 12 & & 31 & 8 & \\
\hline No & 174 & 64 & 110 & & 114 & 60 & & 110 & 64 & & 137 & 37 & \\
\hline \multicolumn{2}{|l|}{ Vascular invasion } & & & 0.307 & & & 0.554 & & & 0.337 & & & 0.278 \\
\hline Yes & 35 & 10 & 25 & & 25 & 10 & & 25 & 10 & & 30 & 5 & \\
\hline No & 178 & 67 & 111 & & 118 & 60 & & 112 & 66 & & 138 & 40 & \\
\hline \multicolumn{3}{|c|}{ Mucinous adenocarcinoma } & & 0.707 & & & 0.831 & & & 0.124 & & & 0.897 \\
\hline Yes & 20 & 8 & 12 & & 13 & 7 & & 16 & 4 & & 16 & 4 & \\
\hline No & 193 & 69 & 124 & & 130 & 63 & & 121 & 72 & & 152 & 41 & \\
\hline Differentiation & & & & 0.461 & & & 0.538 & & & 0.739 & & & 0.284 \\
\hline Well & 76 & 25 & 51 & & 49 & 27 & & 50 & 26 & & 63 & 13 & \\
\hline Moderate/Poor & 137 & 52 & 85 & & 94 & 43 & & 87 & 50 & & 105 & 32 & \\
\hline
\end{tabular}

$L D H$, lactic dehydrogenase; FMN, fructosamine; FBG, fasting blood glucose; OS, overall survival. Bold values mean significant $p$ value which is < 0.05 .

\section{Correlation Between B7-H3 and FBG, LDH, or FMN Expression in CRC}

To determine whether B7-H3 expression associated with FBG, LDH, or FMN expression in CRC, correlations analysis was conducted. However, there was no significant linear relationship between the expression of $\mathrm{B} 7-\mathrm{H} 3$ and $\mathrm{FBG}$, LDH or FMN in all CRC tissue. Further, we analyzed the correlation between $\mathrm{B} 7-\mathrm{H} 3$ and $\mathrm{FBG}, \mathrm{LDH}$ or FMN in different stages. We found a linear correlation between B7-H3 and FBG with depth of tumor invasion (T3/4) $(p=0.037, r=0.259)$, lymph node metastasis (N0) $(p=0.004, r=0.259)$ and TNM stage $(\mathrm{I} / \mathrm{II})(p=0.009$, $r=0.242$ ) (Figure 3). These findings indicated a significant correlation between the expression of $\mathrm{B} 7-\mathrm{H} 3$ and $\mathrm{FBG}$ in the early stage of CRC.

\section{Prognostic Value of B7-H3, FBG, LDH, and FMN Expression}

OS was computed from the date of surgery until the patient's death. The 5-year survival rate was $63.4 \%$ (135/213) for patients with CRC in this study. We respectively analyzed the associations between OS and the expression of B7-H3, FBG, LDH, or FMN. Although the expression of $\mathrm{B} 7-\mathrm{H} 3$ did not affect the OS of patients $(p=0.195)$ (Figure 4A), notably, patients with the high expression of FBG, LDH, or FMN exhibited a significantly worse OS compared to patients with low expression $(p=0.004$, 


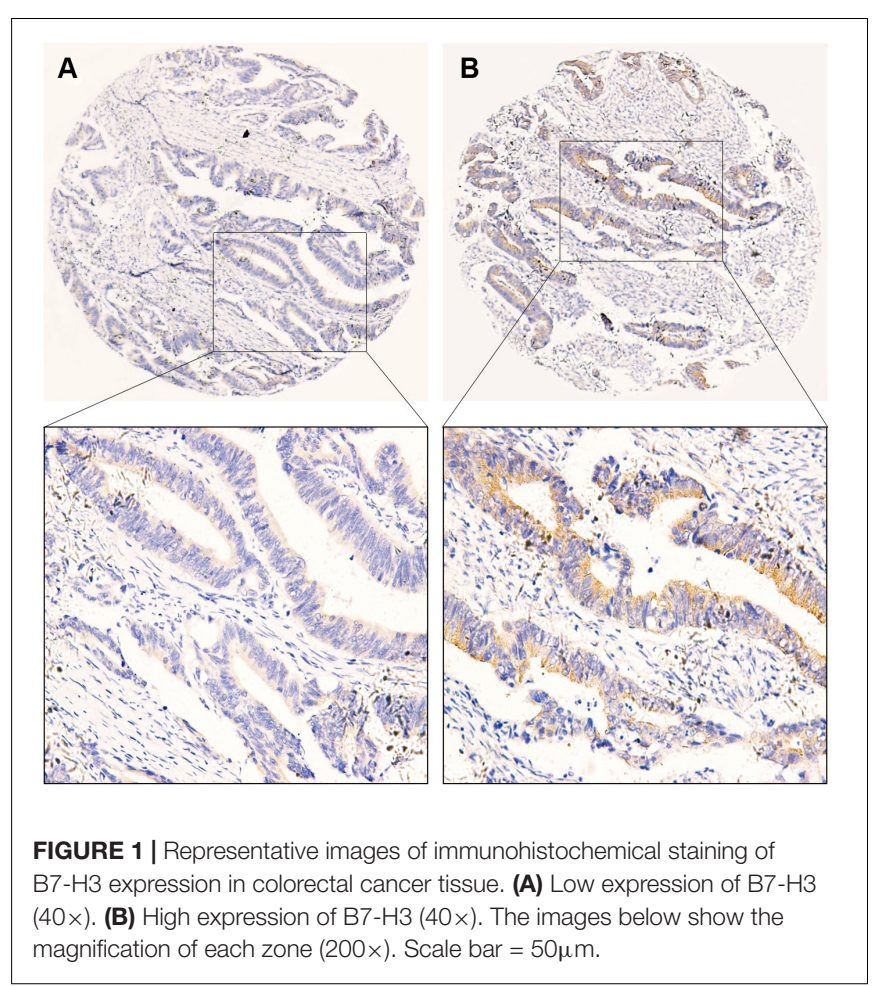

$p=0.009$, and $p=0.001$ ) (Figures 4B-D). Furthermore, we also evaluated the correlation between OS and FBG, LDH, or FMN in the subgroup with different expression of B7-H3. Interestingly, in the subgroup with high B7-H3 expression, the only subgroup with high FMN expression exhibited significantly worse OS compared to the subgroup with high FBG or LDH expression ( $p=0.028$ ) (Figures 4E,G,I). However, a subgroup of patients with low expression of B7-H3 and high FBG, or high LDH, or high FMN exhibited significantly worse prognosis as compared to patients with low expression of FBG, LDH or FMN ( $p=0.002$, $p<0.0001$, and $p=0.014$ ) (Figures 4F,H,J).

Furthermore, to evaluate the risk factors associated with the prognosis of patients with CRC, univariate Cox proportional hazard model analysis was performed. As represented in Table 2, depth of tumor invasion (T3/4), lymph node metastasis (N1/2), distant metastasis, TNM stage (III/IV), neural invasion, high expression of FBG, high expression of $\mathrm{LDH}$, high expression of FMN, high expression of both B7-H3 and FMN, low expression of B7-H3 and high expression of FBG, low expression of B7$\mathrm{H} 3$ and high expression of $\mathrm{LDH}$, low expression of $\mathrm{B} 7-\mathrm{H} 3$ and high expression of FMN were highly correlated with the OS of patients with CRC. Besides, the multivariate analysis revealed that distant metastasis and high expression of FBG were significant independent prognostic factors for OS of patients with CRC (Table 2).

\section{Development and Validation of Nomograms for Predicting Prognosis}

To envisage the prognostic significance of B7-H3, FBG, LDH, and FMN expression, we generated nomograms for OS based on the expression of B7-H3, FBG, LDH, and FMN, and other clinicopathological characteristics (Figure 5A). Using nomogram construction, we could identify the score on the point-scale corresponding to significant factors of each subtype. Then, the total points of each patient were calculated by adding up the score of independent variables altogether. Lastly, by analyzing the complete point scale, we were able to estimate the probability of survival at different time points. The findings indicated that distant metastasis was a significant determinant of prognosis, followed by the FBG expression, depth of tumor invasion, and TNM stage. Overall, it implied that, the larger the score, the higher the survival rate, and vice versa. Subsequently, we were able to predict the prognosis based on nomogram analysis. Figures 5B,C showed the 3-year and 5-year calibration of the nomogram for OS, establishing a pronounced prediction accuracy of this nomogram, indicating that calibration curves for nomogram revealed no deviations from the reference line.

\section{DISCUSSION}

Colorectal cancer remains one of the most frequently diagnosed malignancies worldwide. Increasing pieces of evidence support that the majority of CRC are sporadic, which are predominantly attributable to the constellation of modifiable risk factors characterizing westernization as the significant risk factor (19). These risk factors have also been consistently related to the occurrence of DM (20). In addition, the risk of developing CRC was estimated to be $27 \%$ higher in patients with type $2 \mathrm{DM}$ compared to non-diabetic controls (21). Recently, a meta-analysis by Zhu et al. suggested that diabetes had a negative effect on CRC in OS (22). DM was a chronic disease mainly characterized by disturbances in glucose metabolism (23). Besides, altered energy metabolism is one of the biochemical fingerprints of cancer cells, representing it as one of the hallmarks of cancer, including CRC. Colorectal cancer has also been characterized by altered glucose metabolism mediated by glycolytic pathways (24). Although conceptual advances have significantly improved the understanding on the clinical significance of tumor metabolism, only limited studies have been focused on B7-H3 as indicators of glucose metabolism in CRC patients. Therefore, this study investigated the survival outcome of patients with CRC by the indicators of glucose metabolism.

In the present study, three clinical indicators most frequently used in our hospital were selected, including FBG, LDH, and FMN. FBG was measured as the major criterion of DM, indicating the level of glucose in the blood. $\mathrm{LDH}$ is a key enzyme of glycolysis, which catalyzes the inter-conversion of pyruvate and lactate, and it also converts pyruvate, the final product of glycolysis, to lactate in the absence of oxygen. The high expression of LDH can reveal abnormal glucose metabolism. Fructosamine is formed through glycosylation of serum protein, such as albumin. The concentration of FMN in serum directly reflects blood glucose concentration precisely than $\mathrm{HbAlc}$.

Furthermore, a deregulated or altered energy metabolism has been predominantly recognized as the "hallmarks of cancer." Recently, studies have implicated different roles of lactate 

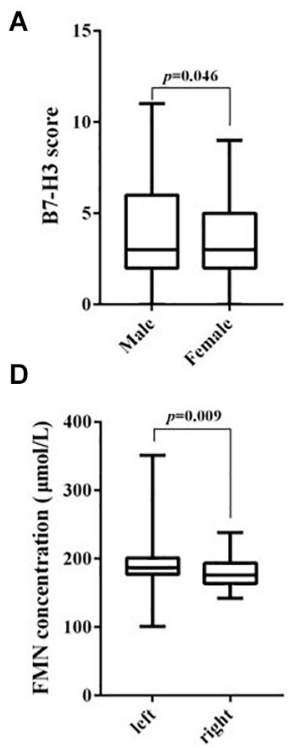

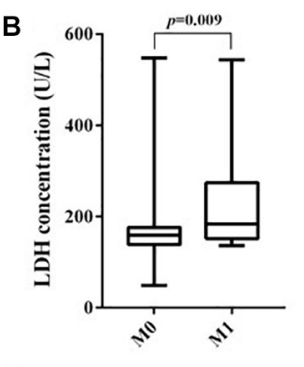

E

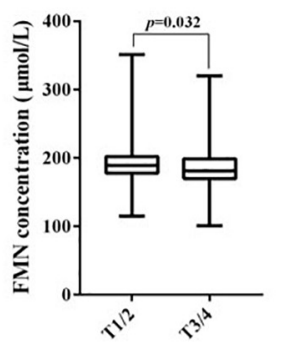

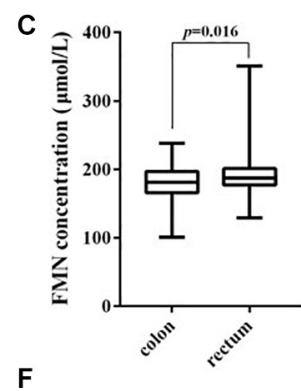

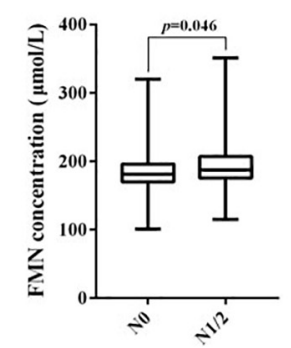

G

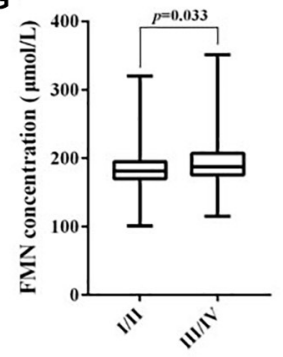

FIGURE 2 | Correlation between B7-H3, LDH, and FMN expression and clinicopathological parameters. (A) B7-H3 and gender; (B) LDH and distant metastasis; (C) FMN and tumor location; (D) FMN and colon cancer site; (E) FMN and depth of tumor invasion; (F) FMN and lymph node metastasis; (G) FMN and TNM stage.
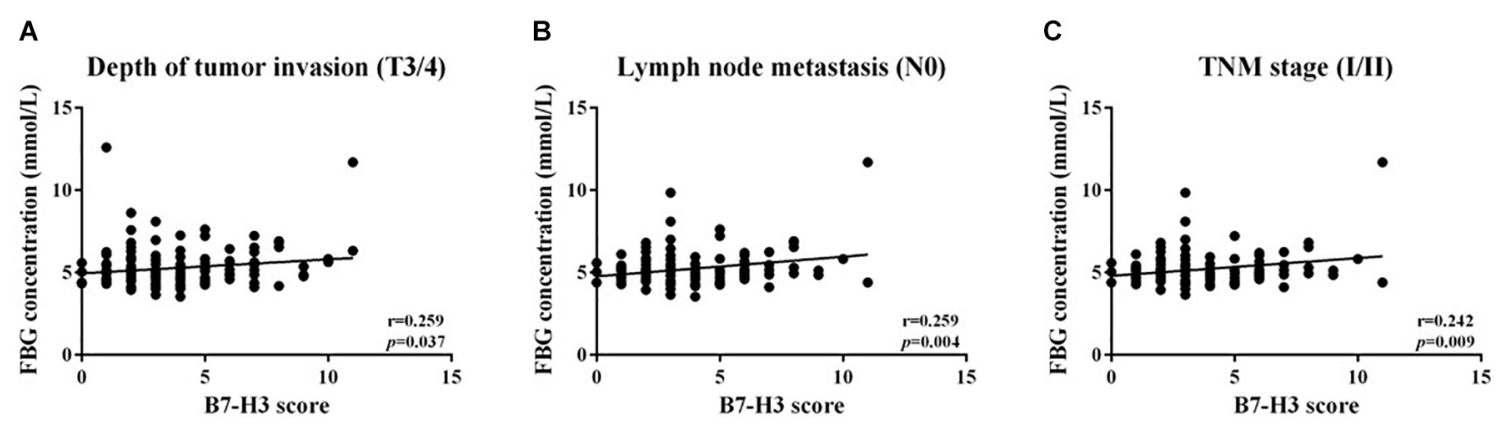

FIGURE 3 | Positive linear relationship between B7-H3 and FBG. (A) Depth of tumor invasion (T3/4); (B) lymph node metastasis (NO); (C) TNM stage (I/II).

export/import contributing to the survival and growth of cancer. In this study, the expression of $\mathrm{LDH}$ was significantly correlated with tumor location and distant metastasis, the expression of FMN was notably associated with lymph node metastasis and TNM stage; however, no significant relationship between the expression of FBG and clinicopathological characteristics were recorded. Next, a significant correlation between $\mathrm{LDH}$ expression and distant metastasis, also between FMN expression and tumor location, colon cancer site, depth of tumor invasion, lymph node metastasis, and TNM stage was demonstrated with Mann Whitney test. In contrast, FBG expression had no relation with pathological features. These findings suggested the respective expression of LDH or FMN had a closer relationship with the parameters of CRC patients than FBG expression in this study. Moreover, high expression of FBG, LDH, or FMN all indicated poor OS of CRC patients eloquently. Conceivably, FBG, LDH, and FMN could predict the prognosis of patients with CRC as an independent prognostic factor. Clinically, the value of FBG,
LDH, and FMN could be controlled to perceive the progression in patients with CRC.

Treatment with immune checkpoint inhibitors has emerged as a frontline treatment for patients with CRC, mainly targeting cytotoxic T-lymphocyte antigen 4 (CTLA-4) and programed death-1 receptor (PD1) and its ligand PD-L1 (25). B7-H3, as one of the crucial immune checkpoint proteins, plays a critical role in the occurrence and development of CRC. Clinically, the expression of B7-H3 was associated with unfavorable outcomes in CRC patients $(12,26)$. However, there was no relationship between B7-H3 expression and OS in this study, which might be attributed to low sample size or the uncertainty of repeated experiments. Nevertheless, over 1000 cases were included in other investigations in order to predict the prognosis of patients. It is noteworthy that the overexpression of $\mathrm{B} 7-\mathrm{H} 3$ has been suggested as a predictor of poor OS in our previous study with more than 200 patients. Thus, the prognostic significance of B7-H3 warrants further validation in future investigation. 

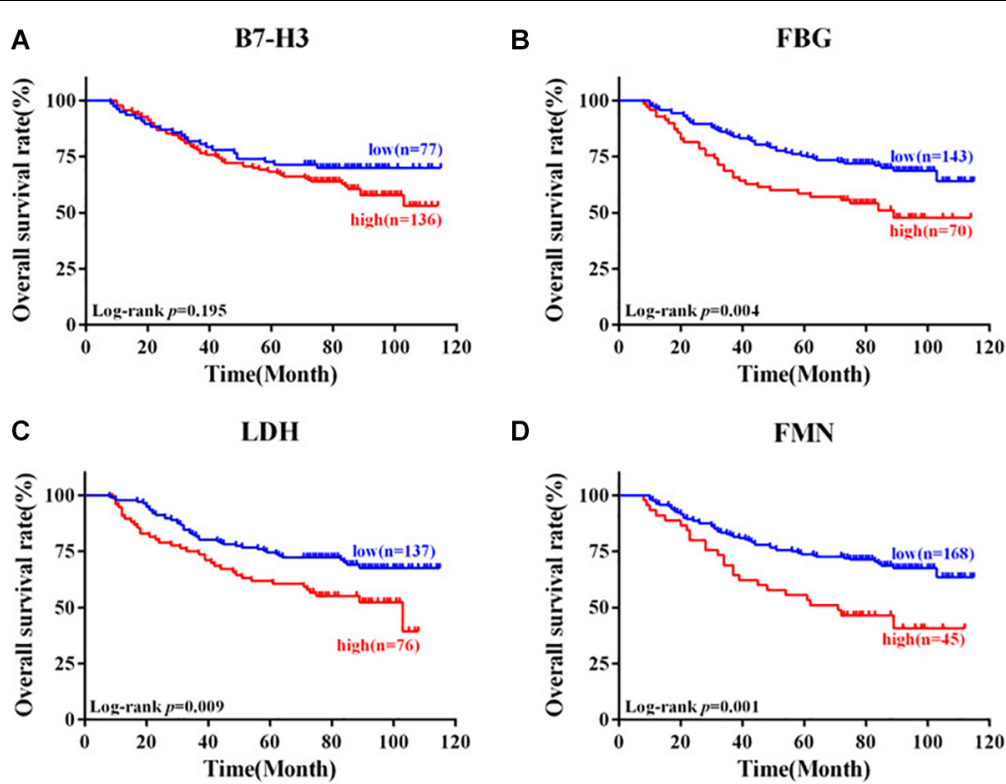

D

FMN

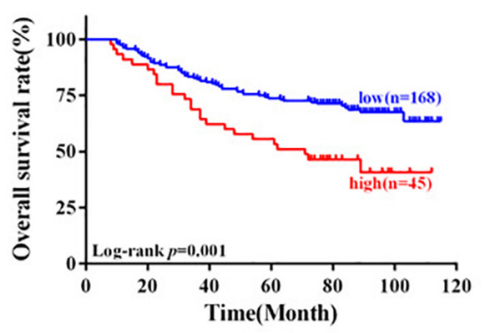

E Subgroup of B7-H3 high expression

$\mathbf{F}$

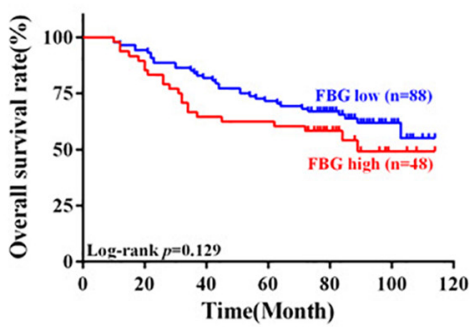

G

Subgroup of B7-H3 high expression

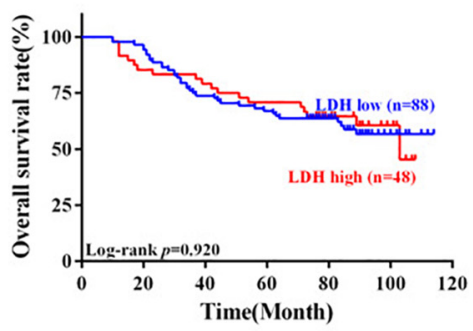

H

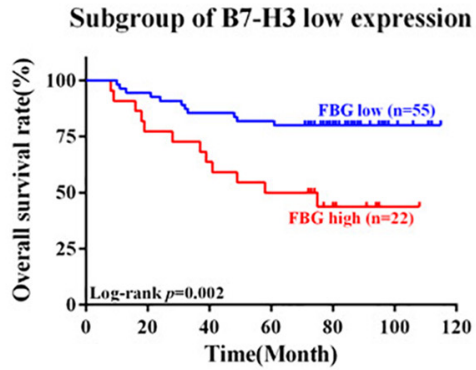

Subgroup of B7-H3 low expression

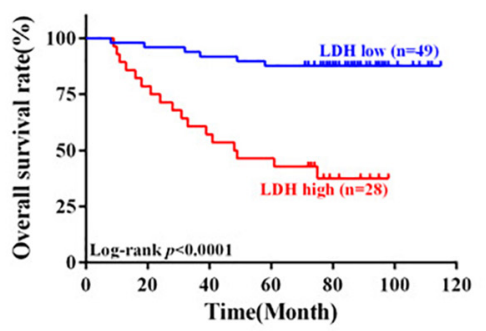

I Subgroup of B7-H3 high expression

$\mathbf{J}$

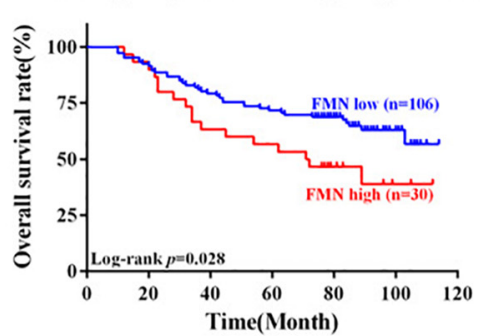

Subgroup of B7-H3 low expression

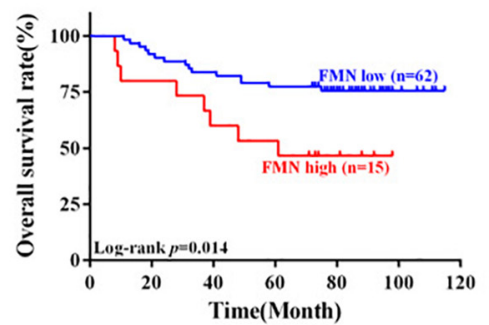

FIGURE 4 | Kaplan-Meier survival curves of patients with colorectal cancer expressing B7-H3, FBG, LDH, and FMN. (A) Patients with low expression of B7-H3 vs. high expression of B7-H3. (B) Patients with low expression of FBG vs. high expression of FBG. (C) Patients with low expression of LDH vs. high expression of LDH. (D) Patients with low expression of FMN vs. high expression of FMN. (E) Subgroup of patients with high expression of B7-H3 and low expression of FBG vs. high expression of FBG. (F) Subgroup of patients with low expression of B7-H3 and low expression of FBG vs. high expression of FBG. (G) Subgroup of patients with high expression of $\mathrm{B} 7-\mathrm{H} 3$ and low expression of $\mathrm{LDH}$ vs. high expression of $\mathrm{LDH}$. (H) Subgroup of patients with low expression of B7-H3 and low expression of LDH vs. high expression of $\mathrm{LDH}$. (I) Subgroup of patients with high expression of B7-H3 and low expression of FMN vs. high expression of FMN. (J) Subgroup of patients with low expression of $\mathrm{B} 7-\mathrm{H} 3$ and low expression of FMN vs. high expression of FMN. 
TABLE 2 | Univariate and multivariate analyses of clinical parameters associated with OS in CRC patients.

Clinical parameter

Gender (male vs. female)
Age ( $\geq 60$ vs. $<60$ )

Tumor location (colon vs. rectum)

Colon cancer site (right-sided vs. left-sided)

Depth of tumor invasion (T3/4 vs. T1/2)

Lymph node metastasis (N1/2 vs. NO)

Distant metastasis (yes vs. no)

TNM stage (III/IV vs. I/II)

Neural invasion (yes vs. no)

Vascular invasion (yes vs. no)

Mucinous adenocarcinoma (no vs. yes)

Differentiation (well vs. moderate/poor)

B7-H3 (high vs. low)

FBG (high vs. low)

LDH (high vs. low)

FMN (high vs. low)

B7-H3 high (FBG high vs. low)

$\mathrm{B} 7-\mathrm{H} 3$ high (LDH high vs. low)

$\mathrm{B} 7-\mathrm{H} 3$ high (FMN high vs. low)

B7-H3 low (FBG high vs. low)

B7-H3 low (LDH high vs. low)

B7-H3 low (FMN high vs. low)
Age $(\geq 60$ vs. $<60)$

Univariate analysis

Bold values mean significant $p$ value which is $<0.05$.

\begin{tabular}{|c|c|c|c|}
\hline \multicolumn{2}{|c|}{ Univariate analysis } & \multicolumn{2}{|c|}{ Multivariate analysis } \\
\hline HR $(95 \% \mathrm{Cl})$ & $p$ value & HR $(95 \% \mathrm{Cl})$ & $p$ value \\
\hline $0.876(0.559-1.373)$ & 0.563 & & \\
\hline $1.201(0.753-1.916)$ & 0.441 & & \\
\hline $0.770(0.492-1.206)$ & 0.254 & & \\
\hline $1.112(0.624-1.983)$ & 0.719 & & \\
\hline $2.481(1.367-4.503)$ & 0.003 & $1.812(0.972-3.372)$ & 0.061 \\
\hline $2.445(1.547-3.865)$ & 0.000 & $0.597(0.177-2.015)$ & 0.406 \\
\hline 11.646 (6.364-21.312) & 0.000 & $5.949(2.788-12.697)$ & 0.000 \\
\hline $3.001(1.871-4.814)$ & 0.000 & $3.349(0.911-12.307)$ & 0.069 \\
\hline $1.726(1.037-2.871)$ & 0.036 & $1.219(0.699-2.126)$ & 0.484 \\
\hline $1.565(0.914-2.681)$ & 0.103 & & \\
\hline $0.792(0.344-1.824)$ & 0.584 & & \\
\hline $0.786(0.500-1.236)$ & 0.297 & & \\
\hline $1.377(0.846-2.240)$ & 0.198 & & \\
\hline $1.916(1.223-3.000)$ & 0.005 & $1.733(1.050-2.861)$ & 0.032 \\
\hline $1.801(1.153-2.813)$ & 0.010 & $1.430(0.902-2.266)$ & 0.128 \\
\hline $2.154(1.336-3.472)$ & 0.002 & 1.377 (0.807-2.352) & 0.241 \\
\hline $1.444(0.841-2.479)$ & 0.183 & & \\
\hline $0.972(0.557-1.695)$ & 0.920 & & \\
\hline $1.881(1.059-3.339)$ & 0.031 & & \\
\hline 3.393 (1.493-7.709) & 0.004 & & \\
\hline 7.107 (2.785-18.138) & 0.000 & & \\
\hline $2.800(1.184-6.625)$ & 0.019 & & \\
\hline
\end{tabular}

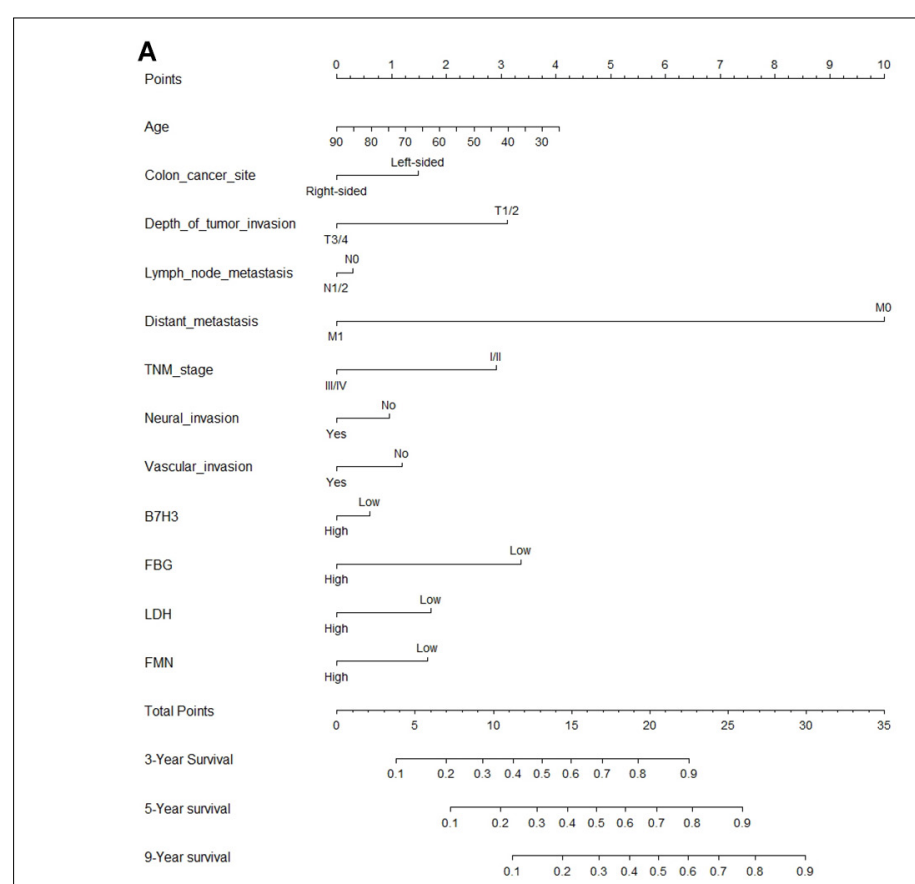

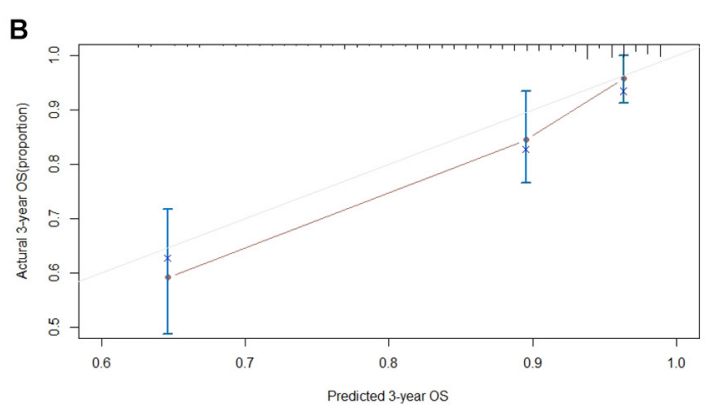

C

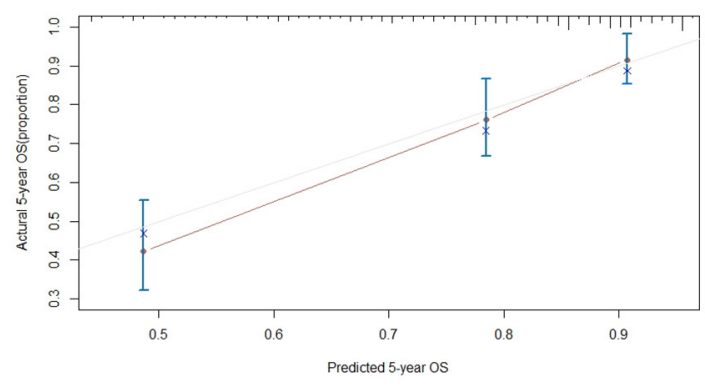

FIGURE 5 | Nomogram based prediction of prognosis. (A) Nomogram for OS based on B7-H3, FBG, LDH, FMN, and other clinicopathological characteristics. (B) The calibration of the nomogram for OS of 3 years. (C) The calibration of nomogram for OS of 5-year. 
Therefore, B7-H3 was still a feasible and effective marker to predict the prognosis in patients with CRC. Besides, recently, B7$\mathrm{H} 3$ antibodies had been reported to be safely used in humans in early phase clinical trials $(27,28)$. Therefore, B7-H3 might serve as a reliable biomarker and therapeutic target for CRC.

Previously, our team has analyzed the non-immunological role of B7-H3 in CRC tumorigenesis, which mainly focused on anti-apoptosis (29), pro-metastasis $(30,31), 5-\mathrm{Fu}$ resistance $(32,33)$, and lipid metabolism (34); however, the relationship between B7-H3 and glucose metabolism was not investigated. Recently, Shi et al. suggested that B7-H3 might be a novel regulator of glucose metabolism via regulating $\mathrm{HK} 2$ expression in CRC cells (14). It motivated us to explore the clinical and prognostic significance of $\mathrm{B} 7-\mathrm{H} 3$ expression as indicators of glucose metabolism in patients with CRC. Thus, we evaluated the clinical correlation and performed survival analysis in patients with CRC for the combined expression of B7-H3 with FBG, $\mathrm{LDH}$, and FMN. We found a linear relationship between B7-H3 and FBG with the depth of tumor invasion (T3/4), lymph node metastasis (N0), and TNM stage (I/II). However, there were no significant linear relationships between the expression of B7-H3 and FBG, LDH, or FMN in all CRC tissue, and also between B7$\mathrm{H} 3$ and LDH or FMN among different subgroups. These results indicated a positive correlation between the expression of B7$\mathrm{H} 3$ and FBG in the early stage of CRC tissue. Furthermore, we considered analyzing the risk of CRC by investigating the aberrant expression of $\mathrm{B} 7-\mathrm{H} 3$ and $\mathrm{FBG}$. In the future, we anticipate detecting B7-H3 and FBG in biopsy tissue specimens and serum of patients with CRC. However, it would possibly be beneficial if the expression of $\mathrm{B} 7-\mathrm{H} 3$ could also be determined in the serum of patients. Further, a subgroup of B7-H3 high expression revealed that only patients with high FMN expression exhibited significantly worse OS compared with FBG and LDH expression. However, a subgroup of patients with low expression of B7-H3 and high FBG, or high LDH, or high FMN exhibited significantly worse prognosis as compared to patients with low expression of FBG, LDH, or FMN. These shreds of evidence implied that FMN is a crucial factor for OS prediction in CRC patients irrespective of high or low expression of B7H3. Besides, high expression FBG or $\mathrm{LDH}$ could warn poor survival outcomes when B7-H3 expression was low. Possibly, in the subgroup of patients with low expression of $\mathrm{B} 7-\mathrm{H} 3$, indicators of glucose metabolism (FBG, LDH, and FMN) should be monitored continually for improved evaluation of prognosis in these patients. Moreover, Cox regression analysis and nomogram manifested the contribution of different clinicopathological characteristics to OS. Conceivably, the survival status and rate

\section{REFERENCES}

1. Siegel RL, Miller KD, Jemal A. Cancer statistics, 2019. CA Cancer J Clin. (2019) 69:7-34.

2. Chen W, Zheng R, Baade PD, Zhang S, Zeng H, Bray F, et al. Cancer statistics in China, 2015. CA Cancer J Clin. (2016) 66:115-32. doi: 10.3322/caac.21338

3. Gu MJ, Huang QC, Bao CZ, Li YJ, Li XQ, Ye D, et al. Attributable causes of colorectal cancer in China. BMC Cancer. (2018) 18:38. doi: 10.1186/s12885017-3968-z of CRC patients could be estimated to stratify the patients for clinical treatment appropriately.

The present study revealed the clinical and prognostic significance of $\mathrm{B} 7-\mathrm{H} 3$ and the clinical indicators of glucose metabolism (FBG, LDH, and FMN) in patients with CRC. Collectively, the findings of this study presented $\mathrm{B} 7-\mathrm{H} 3$ as a hub molecular biomarker, which, combined with indicators like FBG, $\mathrm{LDH}$, or FMN, could possibly reflect the clinical outcomes of patients with CRC. B7-H3 intervention and control of glucose metabolic level appeared to be a promising antitumor strategy with possible benefits in clinical translation. In conclusion, this study provided new insight into the relationship of B7-H3 and glucose metabolism in patients with CRC.

\section{DATA AVAILABILITY STATEMENT}

All datasets presented in this study are included in the article/supplementary material.

\section{ETHICS STATEMENT}

The studies involving human participants were reviewed and approved by Medical Ethics Committee of the Affiliated Hospital of Jiangnan University. The patients/participants provided their written informed consent to participate in this study. Written informed consent was obtained from the individual(s) for the publication of any potentially identifiable images or data included in this article.

\section{AUTHOR CONTRIBUTIONS}

TZ, XQ, YM, and DH designed the research. TZ, YJ, XJ, and LL performed the research. TZ and LL analyzed the data. TZ, YJ, $\mathrm{XJ}$, and LL wrote the manuscript. XQ, YM, and DH revised the manuscript. All authors contributed to the article and approved the submitted version.

\section{ACKNOWLEDGMENTS}

We gratefully acknowledge the financial support from the National Natural Science Foundation Youth Project of China (81902492), the Youth Project of Wuxi Health Committee (Q201822), and Natural Science Foundation of Jiangsu Province (BK20171150).

4. $\mathrm{Hu} \mathrm{C}$, Jia W. Therapeutic medications against diabetes: what we have and what we expect. Adv Drug Deliv Rev. (2019) 139:3-15. doi: 10.1016/j.addr.2018.11. 008

5. Zheng Y, Ley SH, Hu FB. Global aetiology and epidemiology of type 2 diabetes mellitus and its complications. Nat Rev Endocrinol. (2018) 14:88-98. doi: 10.1038/nrendo.2017.151

6. Tsilidis KK, Kasimis JC, Lopez DS, Ntzani EE, Ioannidis JP. Type 2 diabetes and cancer: umbrella review of meta-analyses of observational studies. BMJ. (2015) 350:g7607. doi: 10.1136/bmj.g7607 
7. Park H, Cho S, Woo H, Park SK, Shin HR, Chang SH, et al. Fasting glucose and risk of colorectal cancer in the Korean Multi-center Cancer Cohort. PLoS One. (2017) 12:e0188465. doi: 10.1371/journal.pone.0188465

8. Graziano F, Ruzzo A, Giacomini E, Ricciardi T, Aprile G, Loupakis F, et al. Glycolysis gene expression analysis and selective metabolic advantage in the clinical progression of colorectal cancer. Pharmacogenomics J. (2017) 17:25864. doi: 10.1038/tpj.2016.13

9. Wang Y, Lu JH, Wu QN, Jin Y, Wang DS, Chen YX, et al. LncRNA LINRIS stabilizes IGF2BP2 and promotes the aerobic glycolysis in colorectal cancer. Mol Cancer. (2019) 18:174.

10. Deng F, Zhou R, Lin C, Yang S, Wang H, Li W, et al. Tumor-secreted dickkopf2 accelerates aerobic glycolysis and promotes angiogenesis in colorectal cancer. Theranostics. (2019) 9:1001-14. doi: 10.7150/thno.30056

11. Castellanos JR, Purvis IJ, Labak CM, Guda MR, Tsung AJ, Velpula KK, et al. B7-H3 role in the immune landscape of cancer. Am J Clin Exp Immunol. (2017) 6:66-75.

12. Tang J, Jiang W, Liu D, Luo J, Wu X, Pan Z, et al. The comprehensive molecular landscape of the immunologic co-stimulator B7 and TNFR ligand receptor families in colorectal cancer: immunotherapeutic implications with microsatellite instability. Oncoimmunology. (2018) 7:e1488566. doi: 10.1080/ 2162402x.2018.1488566

13. Fang C, Li S, Xu R, Guo H, Jiang R, Ding S, et al. Soluble B7-H3 (sB7-H3) is over-expressed in the serum of type 1 diabetes patients. Diabetes Res Clin Pract. (2018) 143:332-6. doi: 10.1016/j.diabres.2018.08.004

14. Shi T, Ma Y, Cao L, Zhan S, Xu Y, Fu F, et al. B7-H3 promotes aerobic glycolysis and chemoresistance in colorectal cancer cells by regulating HK2. Cell Death Dis. (2019) 10:308.

15. Wei Y, Xu H, Dai J, Peng J, Wang W, Xia L, et al. Prognostic significance of serum lactic acid, lactate dehydrogenase, and albumin levels in patients with metastatic colorectal cancer. BioMed Res Int. (2018) 2018:1804086.

16. Zhao Z, Han F, Yang S, Hua L, Wu J, Zhan W. The clinicopathologic importance of serum lactic dehydrogenase in patients with gastric cancer. Dis Markers. (2014) 2014:140913.

17. Misciagna G, De Michele G, Guerra V, Cisternino AM, Di Leo A, Freudenheim JL, et al. Serum fructosamine and colorectal adenomas. Eur J Epidemiol. (2004) 19:425-32. doi: 10.1023/b:ejep.0000027359.95727.24

18. Zhang T, Wang F, Wu JY, Qiu ZC, Wang Y, Liu F, et al. Clinical correlation of B7-H3 and B3GALT4 with the prognosis of colorectal cancer. World J Gastroenterol. (2018) 24:3538-46. doi: 10.3748/wjg.v24.i31.3538

19. Keum N, Giovannucci E. Global burden of colorectal cancer: emerging trends, risk factors and prevention strategies. Nat Rev Gastroenterol Hepatol. (2019) 16:713-32. doi: 10.1038/s41575-019-0189-8

20. Volaco A, Cavalcanti AM, Filho RP, Precoma DB. Socioeconomic status: the missing link between obesity and diabetes mellitus? Curr Diabetes Rev. (2018) 14:321-6. doi: 10.2174/1573399813666170621123227

21. Gonzalez N, Prieto I, Del Puerto-Nevado L, Portal-Nunez S, Ardura JA, Corton M, et al. 2017 update on the relationship between diabetes and colorectal cancer: epidemiology, potential molecular mechanisms and therapeutic implications. Oncotarget. (2017) 8:18456-85. doi: 10.18632/ oncotarget.14472
22. Zhu B, Wu X, Wu B, Pei D, Zhang L, Wei L. The relationship between diabetes and colorectal cancer prognosis: a meta-analysis based on the cohort studies. PLoS One. (2017) 12:e0176068. doi: 10.1371/journal.pone.0176068

23. Wu H, Deng X, Shi Y, Su Y, Wei J, Duan H. PGC-1alpha, glucose metabolism and type 2 diabetes mellitus. J Endocrinol. (2016) 229:R99-115.

24. Wang G, Wang JJ, Yin PH, Xu K, Wang YZ, Shi F, et al. New strategies for targeting glucose metabolism-mediated acidosis for colorectal cancer therapy. J Cell Physiol. (2018) 234:348-68. doi: 10.1002/jcp.26917

25. Ciardiello D, Vitiello PP, Cardone C, Martini G, Troiani T, Martinelli E, et al. Immunotherapy of colorectal cancer: challenges for therapeutic efficacy. Cancer Treat Rev. (2019) 76:22-32. doi: 10.1016/j.ctrv.2019.04.003

26. Fan $\mathrm{H}$, Zhu JH, Yao XQ. Prognostic significance of B7-H3 expression in patients with colorectal cancer: a meta-analysis. Pak J Med Sci. (2016) 32:156873.

27. Majzner RG, Theruvath JL, Nellan A, Heitzeneder S, Cui Y, Mount CW, et al. CAR T cells targeting B7-H3, a pan-cancer antigen, demonstrate potent preclinical activity against pediatric solid tumors and brain tumors. Clin Cancer Res. (2019) 25:2560-74. doi: 10.1158/1078-0432.ccr-18-0432

28. Flem-Karlsen K, Fodstad O, Tan M, Nunes-Xavier CE. B7-H3 in cancer beyond immune regulation. Trends Cancer. (2018) 4:401-4. doi: 10.1016/j. trecan.2018.03.010

29. Zhang T, Jiang B, Zou ST, Liu F, Hua D. Overexpression of B7-H3 augments anti-apoptosis of colorectal cancer cells by Jak2-STAT3. World J Gastroenterol. (2015) 21:1804-13. doi: 10.3748/wjg.v21.i6.1804

30. Jiang B, Zhang T, Liu F, Sun Z, Shi H, Hua D, et al. The co-stimulatory molecule B7-H3 promotes the epithelial-mesenchymal transition in colorectal cancer. Oncotarget. (2016) 7:31755-71. doi: 10.18632/oncotarget.9035

31. Liu F, Zhang T, Zou S, Jiang B, Hua D. B7H3 promotes cell migration and invasion through the Jak2/Stat3/MMP9 signaling pathway in colorectal cancer. Mol Med Rep. (2015) 12:5455-60. doi: 10.3892/mmr.2015.4050

32. Jiang B, Liu F, Liu Z, Zhang T, Hua D. B7-H3 increases thymidylate synthase expression via the PI3k-Akt pathway. Tumour Biol. (2016) 37:9465-72. doi: 10.1007/s13277-015-4740-0

33. Sun ZZ, Zhang T, Ning K, Zhu R, Liu F, Tang SC, et al. B7-H3 upregulates BRCC3 expression, antagonizing DNA damage caused by 5-Fu. Oncol Rep. (2016) 36:231-8. doi: 10.3892/or.2016.4808

34. Wu J, Wang F, Liu X, Zhang T, Liu F, Ge X, et al. Correlation of IDH1 and B7H3 expression with prognosis of CRC patients. Eur J Surg Oncol. (2018) 44:1254-60. doi: 10.1016/j.ejso.2018.05.005

Conflict of Interest: The authors declare that the research was conducted in the absence of any commercial or financial relationships that could be construed as a potential conflict of interest.

Copyright (c) 2020 Zhang, Jin, Jiang, Li, Qi, Mao and Hua. This is an open-access article distributed under the terms of the Creative Commons Attribution License (CC BY). The use, distribution or reproduction in other forums is permitted, provided the original author(s) and the copyright owner(s) are credited and that the original publication in this journal is cited, in accordance with accepted academic practice. No use, distribution or reproduction is permitted which does not comply with these terms. 